\title{
Causes of death following spinal cord injury during inpatient rehabilitation and the first five years after discharge. A Dutch cohort study
}

\author{
R Osterthun ${ }^{1}$, MWM Post ${ }^{2}$, FWA van Asbeck ${ }^{3}$, CMC van Leeuwen ${ }^{2}$ and CF van Koppenhagen ${ }^{3}$
}

Study design: Prospective multicentre cohort study.

Objectives: To determine mortality, causes and determinants of death of individuals with spinal cord injury (SCl) within five years after first inpatient rehabilitation.

Setting: The Netherlands.

Methods: Patients were included on admission to first clinical rehabilitation after traumatic or nontraumatic SCl. Inclusion criteria: age between 18 and 65, American Spinal Injury Association impairment scale A-D and expected long-term wheelchair dependency. Information about survival, cause of death, relevant comorbidity and psychosocial circumstances was obtained from the rehabilitation physician or general practitioner. Determinants of death were retrieved from a prospectively collected database. Deceased persons and survivors were compared using $\chi^{2}$-test and $t$-test. Cox regression analysis was performed to describe independent predictors of death. The Kaplan-Meier method was used to calculate survival curves for independent predictors. Excess mortality was described by a standardized mortality ratio (SMR).

Results: Mean duration of follow up was 6.2 years. A total of 27 persons (12.2\%) died during this period (SMR 5.3). Main causes of death were cardiovascular disease (37.0\%), pulmonary disease $(29.6 \%)$ and neoplasm (14.8\%). Older age at injury, nontraumatic $\mathrm{SCl}$, family history of cardiovascular disease, less social support and a history of other medical conditions on admission were related to death. Older age at injury, nontraumatic $\mathrm{SCl}$ and a history of other medical conditions were independent predictors of death.

Conclusion: Twelve per cent of persons with SCI who had survived the acute hospital phase died during follow up (SMR 5.3). The main causes of death were cardiovascular and pulmonary disease.

Spinal Cord (2014) 52, 483-488; doi:10.1038/sc.2014.28; published online 1 April 2014

Keywords: spinal cord injury; mortality; survival; cause of death

\section{INTRODUCTION}

Although survival of persons with spinal cord injury (SCI) has improved considerably in the last decades, ${ }^{1-3}$ there remains an increased risk of premature death owing to, for example, pneumonia or suicide. ${ }^{1,4-7}$ The main long-term causes of death of persons with SCI have changed from urological complications to cardiovascular and pulmonary diseases. ${ }^{1}$ Several studies on mortality after SCI have been performed. ${ }^{1}$ Comparing results of these studies is however difficult owing to the methodological differences, such as case selection and survival periods. Furthermore, most of these studies restricted their samples to persons with traumatic SCI and most focus on long-term mortality. ${ }^{1,4-7}$ It has been suggested that the decline in mortality since the 1980s is mainly based on improvement of acute care. ${ }^{2,3}$ The risk of death is however still the highest during the first few years after injury. ${ }^{3,7,8}$ In addition to personal and lesion characteristics, several psychosocial and lifestyle factors have been found to be associated with death. ${ }^{4,9,10}$
In the Netherlands, persons with a new SCI are generally admitted to the hospital for a few days to weeks. When their medical condition has been stabilized, they are transferred to a nursing home or a rehabilitation centre with a specialized SCI unit to continue their inpatient rehabilitation. Some information on mortality in the acute care hospital is available, ${ }^{11}$ but post-acute mortality and causes of death of persons with SCI have not previously been described in the Netherlands. Internationally, only limited information is available about the causes of death in the first few years after SCI for persons who survived the hospital phase. ${ }^{5,6,12}$ Understanding the causes of death in this period can yield relevant information for further improvements of care.

The purpose of this study is to describe mortality, causes and determinants of death between the start of first inpatient SCI rehabilitation until five years after discharge in the Netherlands. We hypothesised that besides personal and lesion characteristics, health variables, lifestyle and psychosocial factors are associated with mortality.

${ }^{1}$ Center of Excellence for Rehabilitation Medicine, University Medical Center Utrecht and De Hoogstraat Rehabilitation, Utrecht, The Netherlands; ${ }^{2}$ Brain Center Rudolf Magnus and Center of Excellence for Rehabilitation Medicine, University Medical Center Utrecht and De Hoogstraat Rehabilitation, Utrecht, The Netherlands and ${ }^{3}$ Department of Spinal Cord Management, De Hoogstraat Rehabilitation, Utrecht, The Netherlands

Correspondence: Dr MWM Post, Brain Center Rudolf Magnus and Center of Excellence for Rehabilitation Medicine, University Medical Center Utrecht and De Hoogstraat Rehabilitation, PO Box 85238, 3508 AE Utrecht, The Netherlands.

E-mail: m.post@dehoogstraat.nl

Received 10 August 2013; revised 18 February 2014; accepted 18 February 2014; published online 1 April 2014 


\section{MATERIALS AND METHODS}

\section{Study population}

The study population consisted of 225 individuals with traumatic and nontraumatic SCI included in the Dutch prospective multicentre cohort study 'Restoration of (wheelchair) mobility in SCI rehabilitation. ${ }^{13}$ The observation period started on admission of their first inpatient rehabilitation in eight rehabilitation centres with specialised SCI units in the Netherlands between August 2000 and July 2003. Further inclusion criteria of the cohort were: age between 18 and 65 years, grade A, B, C or D on the American Spinal Injury Association (AISA) Impairment Scale (AIS) and expected wheelchair dependency. Participants were excluded if their SCI was caused by a malignant tumour, if they had a progressive disease, psychiatric problems or insufficient command of the Dutch language to participate in the study.

\section{Procedure}

Information was prospectively collected at the start of active rehabilitation, defined as the moment that the participant was able to sit in a wheelchair for $3-4 \mathrm{~h}$, by a medical examination, an oral interview by a trained research assistant and self-report questionnaires.

Additional information on survival status and, if applicable, cause of death was requested retrospectively from the rehabilitation physician with a form developed for this project. If this procedure yielded insufficient information, the participant's general practitioner was approached.

The research protocol of the cohort study was approved by the Medical Ethics Committee of the SRL/iRv. All persons gave written informed consent. The additional collection of data on survival and causes of death was approved by the local medical ethics committee of De Hoogstraat Rehabilitation, Utrecht, the Netherlands.

\section{Instruments}

Survival status and causes of death: with a structured self-developed form, information was asked about the survival status, cause of death based on the ICD-10, date of death, relevant comorbidity and psychosocial circumstances that had possibly contributed to death.

Lesion characteristics: level and completeness of SCI were assessed according to the International Standards for Neurological Classification of Spinal Cord Injury. ${ }^{14}$ Functional independence was measured with the motor score of the Functional Independence Measure, consisting of 13 items about self-care, mobility, transfers and toileting. The Functional Independence Measure motor score is a responsive measure in persons with SCI. ${ }^{15}$

Psychosocial factors: social support was measured with the Social Support List, which is a reliable and valid short version of the Social Support ListInteractions, consisting of 12 items. ${ }^{16} \mathrm{~A}$ total score is calculated by adding up all items (range 12-48). Mental health and vitality were assessed using the Mental Health and Vitality subscales of the 36-Item Short Form Health Survey (SF-36), consisting of 5 and 4 items, respectively. The SF-36 has shown acceptable reliability and validity in persons with SCI. ${ }^{17}$ Life satisfaction was measured with the LS questions, which have shown good validity in measuring life satisfaction in persons with SCI. ${ }^{18}$

Health factors: whether persons had a history of other medical conditions or a family history of cardiovascular disease was assessed by the research assistant. A history of other medical conditions included all other medical conditions before admission to the rehabilitation centre, other injuries at lesion onset and in-hospital complications. Secondary SCI conditions were not included. Body mass was assessed by the research assistant and body height was self-reported. The Body Mass Index (BMI) was computed and being overweighed was defined as a BMI $>25$.

Lifestyle factors: playing sports before SCI, smoking and drinking alcohol before SCI were self-reported on admission.

\section{Data analysis}

Analyses were performed using IBM SPSS Statistics version 19.0 (Armonk, NY, USA). Differences between deceased persons and survivors were described using the $\chi^{2}$-test and $t$-test. A cox regression analysis was performed to describe independent predictors of death. The Kaplan-Meier method was used to calculate the observed survival curves for independent predictors. Age at injury was dichotomized (age $<45$ years and age $\geqslant 45$ years) to enable the calculation and display of survival curves for this variable. This cut-off point was chosen to create more or less equal-sized groups.

The excess mortality was described by calculating a standardized mortality ratio (SMR). This is the ratio of the observed patient mortality and the expected mortality in the Dutch population. The number of expected deaths during the time interval of the study, adjusted by sex and age, was calculated using the database of Statistics Netherlands (www.cbs.nl).

\section{RESULTS}

\section{General characteristics}

Survival status was successfully identified in 222 persons (98.7\%). The remaining three persons were excluded. Mean time between injury and admission to the rehabilitation centre, that is, time from injury to start of the observation period, was 45.8 (s.d. 43.4) days (median 32 days, range 0-281). Mean age at injury was 40.6 (s.d. 14.1) years (median 40.3 years, range 18.1-65.7), 74.3\% were male, 59.9\% had paraplegia, $69.1 \%$ had a motor complete lesion and $73.0 \%$ had a traumatic lesion. Accidents (69.2\%) and falls (22.2\%) were the most common causes of traumatic SCI. The causes of nontraumatic SCI were vascular disease $(21.7 \%)$, inflammation (15.0\%), iatrogenic $(13.3 \%)$, benign tumour $(10.0 \%)$ and other nontraumatic causes $(40.0 \%)$

A total of 27 persons (12.2\%) died during the mean follow up period of 6.2 (s.d. 1.3) years. With a number of expected deaths in the general population of 5.1, the standardized mortality ratio was 5.3 (95\% CI 3.6-7.6). Mean age at death was 53.5 (s.d. 13.2) years. Mean time between injury and death was 4.1 (s.d. 2.7) years. The survival for persons with traumatic and nontraumatic SCI was $93.2 \%$ and $73.3 \%$ respectively. Mortality rates in the different subgroups of nontraumatic SCI did not significantly differ, although there was a tendency towards higher mortality rates in subgroups with vascular and 'other' nontraumatic causes of SCI. Further characteristics of deceased persons and survivors are displayed in Table 1.

\section{Causes of death}

The characteristics of deceased persons are displayed in Table 2. The main causes of death were cardiovascular disease $(37 \%)$, pulmonary disease (29.6\%) and neoplasm (14.8\%). Three persons (23, 24 and 39 years of age, all with motor complete paraplegia) died during the first year after injury, all from pulmonary embolism, of whom two during inpatient rehabilitation. For one person, the heparin anticoagulation had been restarted a few days before death. The other two persons had used oral anticoagulation during their admission, of which one until death. Seven persons died owing to other cardiovascular disease of which six had a nontraumatic lesion. Of the eight persons who died owing to pulmonary disease, six had a traumatic SCI and five had a tetraplegia. One death occurred owing to homicide. This person had survived a previous assault that had resulted in the SCI. Psychosocial circumstances had possibly contributed to death in $22 \%$ of cases.

\section{Determinants of death}

Older age at injury, nontraumatic cause of injury, family history of cardiovascular disease, less social support and having a history of other medical conditions were related to death (Table 1). Lesion characteristics were not related to death, neither when calculated separately for traumatic and nontraumatic SCI.

In cox regression analyses, age at injury, nontraumatic aetiology and having a history of other medical conditions were independent 
Table 1 Characteristics of deceased persons and survivors

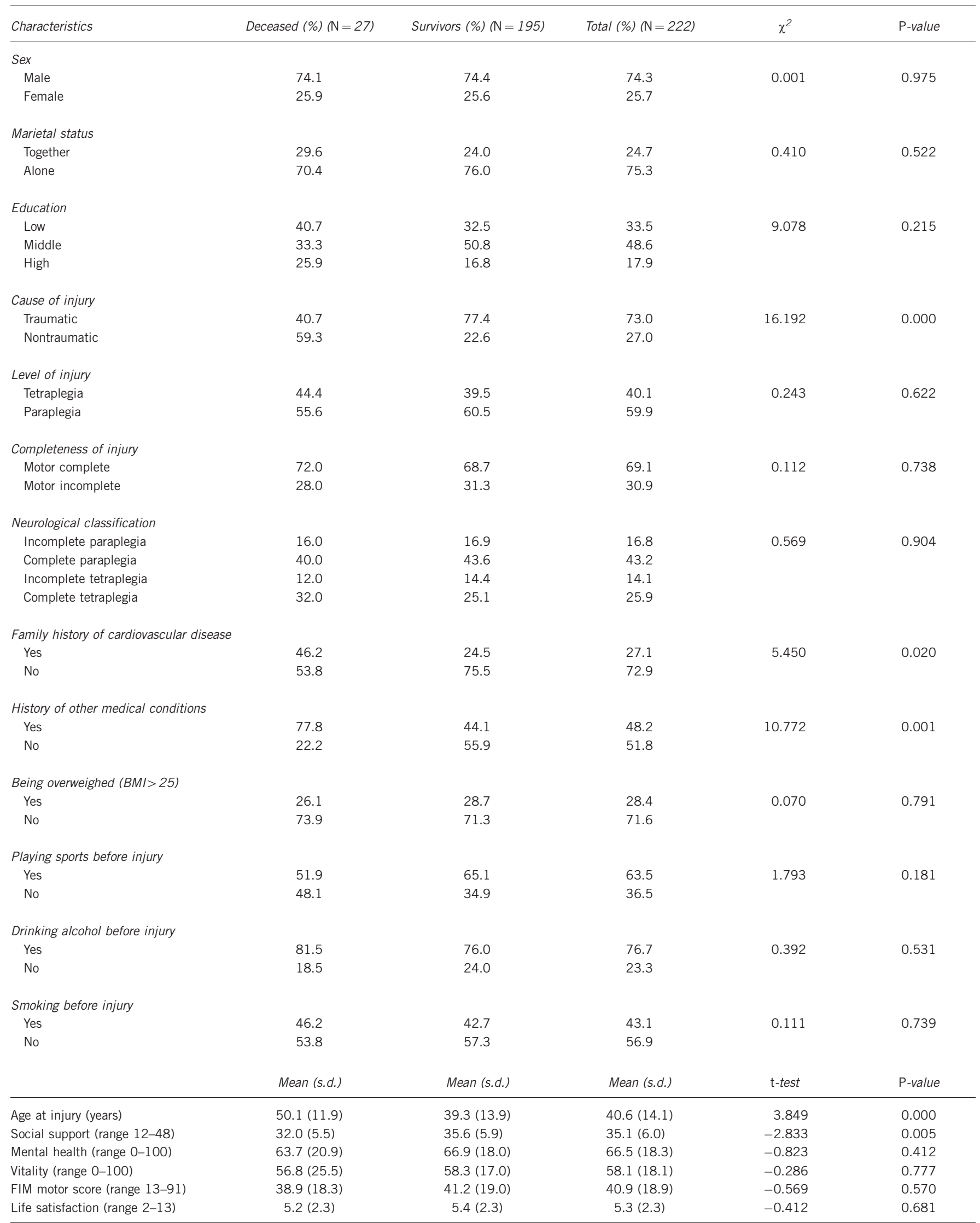

Abbreviations: BMI, body mass index; FIM, Functional Independence Measure. 
Table 2 Characteristics of deceased persons $(N=27)$

\begin{tabular}{|c|c|c|c|c|}
\hline Characteristic & $\begin{array}{c}\text { Traumatic } \\
\text { (N) }\end{array}$ & $\begin{array}{c}\text { Nontraumatic } \\
\text { (N) }\end{array}$ & $\begin{array}{l}\text { Total } \\
\text { (N) }\end{array}$ & $\begin{array}{l}\text { Total } \\
\text { (\%) }\end{array}$ \\
\hline \multicolumn{5}{|l|}{ Cause of death } \\
\hline \multicolumn{5}{|l|}{ Cardiovascular disease } \\
\hline Pulmonary embolism & 2 & 1 & 3 & 11.1 \\
\hline Other cardiovascular disease & 1 & 6 & 7 & 25.9 \\
\hline \multicolumn{5}{|l|}{ Airway/pulmonary } \\
\hline Sepsis airway & 2 & 0 & 2 & 7.4 \\
\hline Influenza and pneumonia & 2 & 2 & 4 & 14.8 \\
\hline Other pulmonary disease & 2 & 0 & 2 & 7.4 \\
\hline Neoplasm & 0 & 4 & 4 & 14.8 \\
\hline Peritonitis & 0 & 1 & 1 & 3.7 \\
\hline Sepsis owing to pressure sore & 1 & 0 & 1 & 3.7 \\
\hline Euthanasia & 0 & 1 & 1 & 3.7 \\
\hline Homicide & 1 & 0 & 1 & 3.7 \\
\hline Unknown & 0 & 1 & 1 & 3.7 \\
\hline \multicolumn{5}{|l|}{ Living situation at moment of death } \\
\hline Independent with partner & & & 13 & 48.1 \\
\hline Independent without partner & & & 4 & 14.8 \\
\hline Independent living with regular help & & & 2 & 7.4 \\
\hline Nursing home & & & 1 & 3.7 \\
\hline Other & & & 5 & 18.5 \\
\hline Unknown/missing & & & 2 & 7.4 \\
\hline \multicolumn{5}{|l|}{ Location of death } \\
\hline Home & & & 12 & 44.4 \\
\hline Hospital & & & 8 & 29.6 \\
\hline Rehabilitation centre & & & 2 & 7.4 \\
\hline Nursing home & & & 2 & 7.4 \\
\hline Other & & & 1 & 3.7 \\
\hline Unknown/missing & & & 2 & 7.4 \\
\hline \multicolumn{5}{|l|}{ Relevant comorbidity } \\
\hline Yes & & & 20 & 74.1 \\
\hline No & & & 4 & 14.8 \\
\hline Unknown/missing & & & 3 & 11.1 \\
\hline \multicolumn{5}{|c|}{ Psychosocial circumstances possibly contributing to death } \\
\hline Yes & & & 6 & 22.2 \\
\hline No & & & 16 & 59.3 \\
\hline Unknown/missing & & & 5 & 18.5 \\
\hline
\end{tabular}

predictors of death (Table 3). Calculated survival curves for independent predictors are shown in Figures 1-3.

\section{DISCUSSION}

A high number of persons with SCI who had survived the acute hospital phase died during the 6.2 year follow up (12\%, standardized mortality ratio 5.3), with cardiovascular and pulmonary diseases as major causes of death (65\%). Age at injury, nontraumatic aetiology and a history of other medical conditions were independent predictors of death.

Comparing the results of our study with other studies is difficult owing to methodological differences. As all participants in our study had survived the acute hospital phase, our results are applicable to the rehabilitation phase and the first few years afterwards. Previously we described an in-hospital mortality rate of $14 \%$ in traumatic SCI. ${ }^{11}$ Considering the generalisation of our results, the participants of this study were on average younger, had more often a complete and traumatic SCI than persons with SCI admitted to Dutch and Flemish rehabilitation centres. ${ }^{19}$ The inclusion criteria of our study thus render a positive selection and, consequently, probably an underestimation of mortality compared with the Dutch SCI population.
Table 3 Cox regression analyses of predictors of death

\begin{tabular}{lccc}
\hline Predictor & Relative risk & $95 \% \mathrm{Cl}$ & $\mathrm{P}$-value \\
\hline Cause of injury (nontraumatic) & 4.0 & $1.6-10.4$ & 0.004 \\
Age at injury (older) & 1.0 & $1.0-1.1$ & 0.046 \\
Having a history of other medical conditions (yes) & 5.7 & $1.9-16.7$ & 0.001 \\
\hline
\end{tabular}

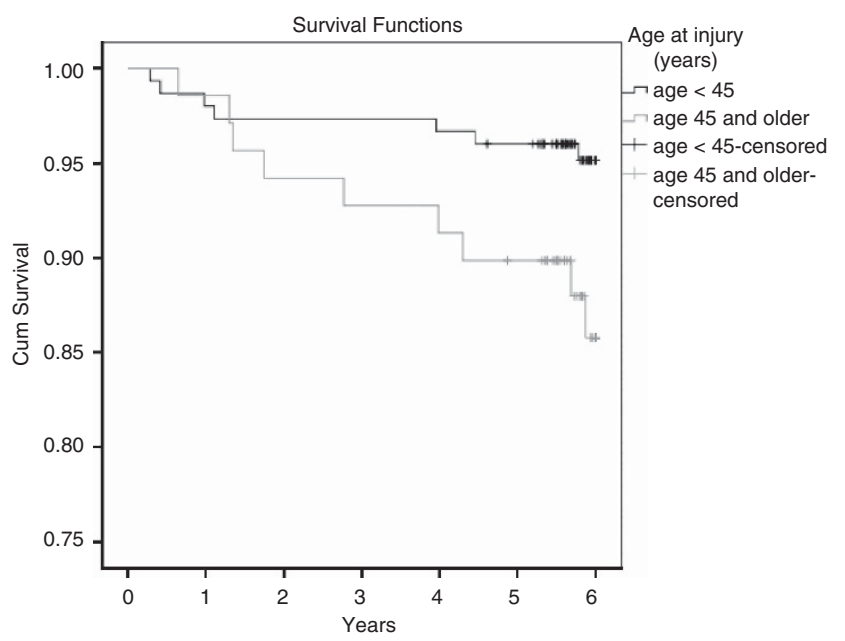

Figure 1 Survival curves for age at injury.

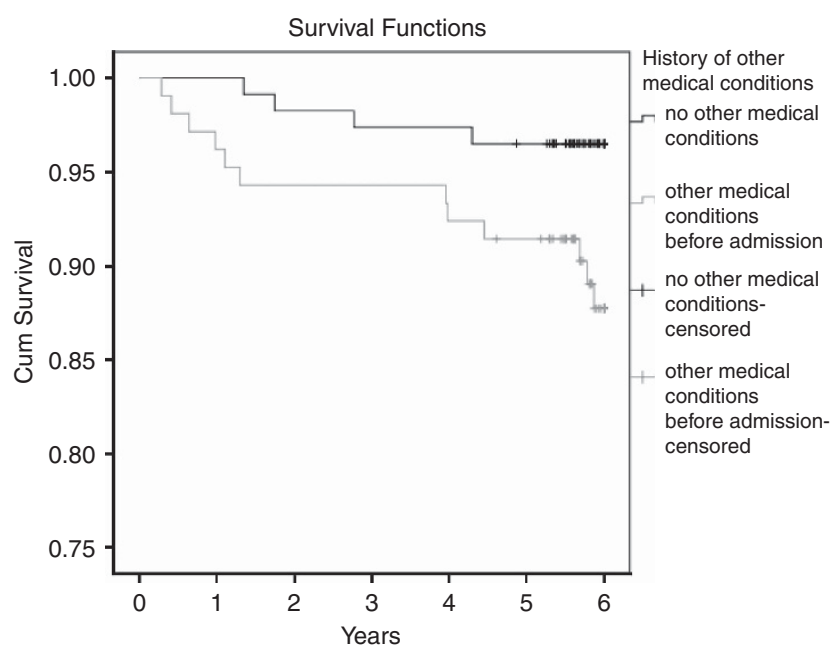

Figure 2 Survival curves for history of other medical conditions.

Hartkopp et al. ${ }^{6}$ described mortality and causes of death of a traumatic SCI population that had also survived the acute phase (days to several months). Our standardized mortality ratio was more than twice as high as in their study, probably owing to the inclusion of nontraumatic SCI in our study. Hartkopp et al. ${ }^{6}$ described a 10-year survival rate of $86.8 \%$, which seems to correlate with our $93.2 \%$ in the subgroup of persons with traumatic SCI after a mean follow up of 6.2 years. Mesard et al. ${ }^{20}$ studied mortality of persons with traumatic SCI between 1955 and 1965. This study found a high mortality rate in the first 3 months and a 5 -year survival of about $91 \%$ for persons who 


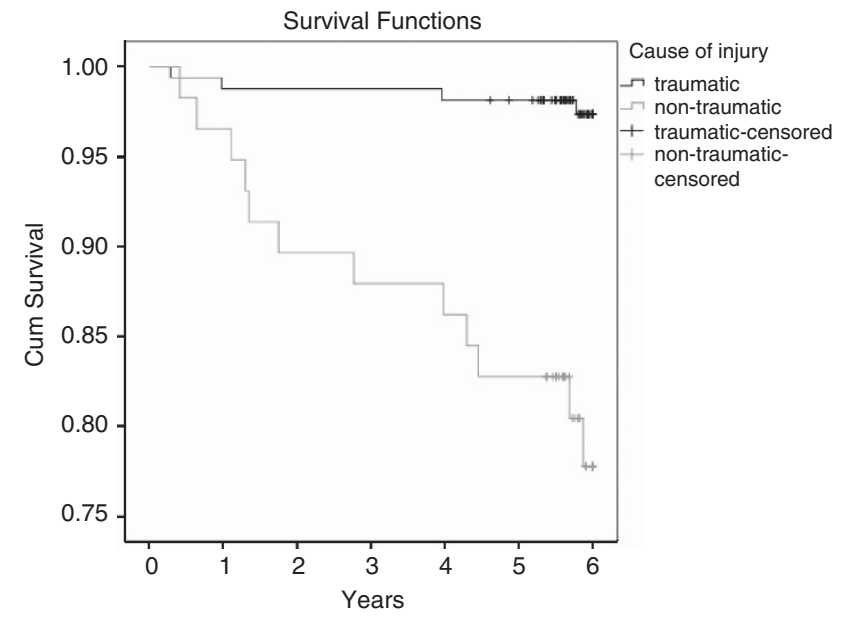

Figure 3 Survival curves for cause of injury.

had survived the first 3 months. ${ }^{20}$ An Israeli study in a large group of persons with nontraumatic SCI described 5-year and 10-year survival rates of 93.7 and $84.2 \%$, respectively. ${ }^{21}$ These are considerably higher than the $73.3 \%$ survival rate of persons with nontraumatic SCI in our study. Unfortunately, there is no consensus on the definition of nontraumatic SCI and outcomes may vary on the underlying disease. , $21^{21}$

Few other studies have compared survival rates in persons with traumatic and nontraumatic SCI. In one study, nontraumatic SCI was a predictor of mortality in univariate analyses, but was not after the adjustment for age. ${ }^{22}$ Another study found no significant differences in risk factors for mortality in nontraumatic and traumatic SCI, other than the effect of age at lesion onset, which was a greater risk factor in the latter group. ${ }^{21}$

Advanced age at injury is commonly described as risk factor of mortality. ${ }^{1}$ The strength of the predictive value of age at injury in our study was reduced in the cox regression analyses by the few young persons who died early after SCI, as can be seen in the survival curve (Figure 1).

A history of other medical conditions was an independent predictor of death in our study. The additional gathered information showed that $74 \%$ had relevant co-morbidities, suggesting this may have been an important factor, which is confirmed by several other studies. ${ }^{1}$

Altogether, persons with nontraumatic SCI are generally older and have more co-morbidities, which makes them even more at risk for premature death. The high mortality ratio in persons with SCI may be partly ascribed to the result of a sedentary lifestyle, causing the cardiovascular system to deteriorate more rapidly than in the general population. A healthy lifestyle thus seems extra important for persons with SCI. ${ }^{23}$

Social support was related to death in bivariate analyses, probably owing to the restricted social support that older people experience. In contrast to several other studies, ${ }^{4,9,10}$ our study did not support the hypothesis that other health, lifestyle and psychosocial factors on admission contributed to death. However, persons with psychiatric diagnosis were excluded, which may have caused a selection bias. Lesion characteristics were also not related to death in our study. Some studies found the same result, ${ }^{5,12,21,22}$ but other studies did find associations with lesion characteristics. $3,4,7,8$
The predominant causes of death we found, pulmonary and cardiovascular disease, are a confirmation of the study by Hartkopp et $a .^{6}$ and other studies that describe long-term mortality in persons with SCI. $4,6,7,22$ No death owing to suicide occurred in our study, whereas in some other studies it appears to be a common cause of death. ${ }^{4-7}$ This may be owing to the exclusion of persons with psychiatric problems in our study. Furthermore, our sample did not include persons with mild lesions, a group that has been reported to have higher risk on suicide. ${ }^{6}$ One death occurred owing to euthanasia. Euthanasia is illegal in the Netherlands. However, it goes without prosecution since 2002 as long as legal requirements are met. We also found no deaths owing to urinary tract complications. Several studies have described a reduction of deaths owing to this cause. ${ }^{1,5,8}$ The improved care and increased awareness of possible urinary tract complications have probably contributed to the absence of urinary tract related deaths in our study.

Pulmonary embolism is described as an early cause of death in older studies. ${ }^{24,25}$ In our study, strikingly, within the first year after the onset of SCI three young people died of a pulmonary embolism. Two of them used oral anticoagulation during their admission, indicating they had a higher risk of developing a thromboembolic event. As one person used oral anticoagulation until death, the dose of oral anticoagulation may not have been correctly adjusted. Nevertheless, this finding implicates the importance of awareness for deep venous thrombosis and pulmonary embolism.

\section{Limitations}

Several limitations should be addressed. In-hospital deaths were not included. The study group was relatively small and concerned a selected cohort of persons with traumatic and nontraumatic SCI. They were expected to remain wheelchair dependent, meaning that persons with mild lesions were excluded. The age criteria for inclusion probably render an underestimation of mortality when compared with SCI-population-based studies. Effects of psychosocial factors on mortality are possibly underestimated as persons with psychiatric problems were excluded.

Nontraumatic SCI may be owing to different aetiologic mechanisms, which makes mortality data in this group more difficult to compare. No firm conclusions can be drawn from our variable 'history of other medical conditions' which included several factors.

\section{Implications}

Persons with nontraumatic SCI, with a higher age at injury and a history of other medical conditions have a higher risk of dying in the first few years after injury. Discussing the importance of a healthy lifestyle with patients in this group should start early and be a routine part of regular follow up after discharge of rehabilitation.

Further research could include a prolonged follow up and a description of in-hospital deaths of persons with SCI in the Netherlands. More research is required on the factors that affect mortality in different nontraumatic etiologies. Awareness of deep venous thrombosis and pulmonary embolism is important to reduce SCI-related deaths.

\section{DATA ARCHIVING}

There were no data to deposit.

\section{CONFLICT OF INTEREST}

The authors declare no conflict of interest. 
1 Van den Berg ME, Castellote JM, de Pedro-Cuesta J, Mahillo-Fernandez I. Survival afte spinal cord injury: a systematic review. J Neurotrauma 2010; 27: 1517-1528.

2 DeVivo MJ. Epidemiology of traumatic spinal cord injury: trends and future implications. Spinal Cord 2012; 50: 365-372.

3 Strauss DJ, DeVivo MJ, Paculdo DR, Brooks J, Paculdo D. Trends in life expectancy after spinal cord injury. Arch Phys Med Rehabil 2006; 87: 1079-1085.

4 Lidal IB, Snekkevik H, Aamodt G, Hjeltnes N, Biering-Sorensen F, Stanghelle JK. Mortality after spinal cord injury in Norway. J Rehabil Med 2007; 39: 145-151.

5 Soden RJ, Walsh J, Middleton JW, Craven ML, Rutkowski SB, Yeo JD. Causes of death after spinal cord injury. Spinal Cord 2000; 38: 604-610.

6 Hartkopp A, Bronnum-Hansen H, Seidenschnur AM, Biering-Sorensen F. Survival and cause of death after traumatic spinal cord injury. A long-term epidemiological survey from Denmark. Spinal Cord 1997; 35: 76-85.

7 Frankel HL, Coll JR, Charlifue SW, Whiteneck GG, Gardner BP, Jamous MA et al. Long-term survival in spinal cord injury: a fifty year investigation. Spinal Cord 1998; 36: 266-274.

8 O'Connor PJ. Survival after spinal cord injury in Australia. Arch Phys Med Rehabil 2005; 86: 37-47.

9 Krause JS, Zhai Y, Saunders LL, Carter RE. Risk of mortality after spinal cord injury: an 8-year prospective study. Arch Phys Med Rehabil 2009; 90: 1708-1715.

10 Cao Y, Krause JS, Dipiro N. Risk factors for mortality after spinal cord injury in the USA. Spinal Cord 2013; 51: 413-418.

11 Van Asbeck FW, Post MW, Pangalila RF. An epidemiological description of spinal cord injuries in the Netherlands in 1994. Spinal Cord 2000; 38: 420-424.

12 Samsa GP, Patrick CH, Feussner JR. Long-term survival of veterans with traumatic spinal cord injury. Arch Neurol 1993; 50: 909-914.

13 De Groot S, Dallmeijer AJ, Post MW, van Asbeck FW, Nene AV, Angenot EL et al. Demographics of the Dutch multicenter prospective cohort study 'restoration of mobility in spinal cord injury rehabilitation'. Spinal Cord 2006; 44 : 668-675.
14 Maynard FM Jr., Bracken MB, Creasey G, Ditunno JF Jr., Donovan WH, Ducker TB. International standards for neurological and functional classification of spinal cord injury. American Spinal Injury Association. Spinal Cord 1997; 35: 266-274.

15 Hall KM, Cohen CE, Wright J, Call M, Werner P. Characteristics of the Functional Independence Measure in traumatic spinal cord injury. Arch Phys Med Rehabil 1999, 80: 1471-1476.

16 Kempen GI, van Eijk LM. The psychometric properties of the SSL-12-I, a short scale for measuring social support in the elderly. Soc Ind Res 1995; 35: 303-312.

17 Forchheimer M, McAweeney M, Tate DG. Use of the SF-36 among persons with spinal cord injury. Am J Phys Med Rehabil 2004; 83: 390-395.

18 Post MW, van Leeuwen CM, van Koppenhagen CF, de Groot S. Validity of the life satisfaction questions, the life satisfaction questionnaire, and the satisfaction with life scale in persons with spinal cord injury. Arch Phys Med Rehabil 2012; 93: 1832-1837.

19 Osterthun R, Post MW, van Asbeck FW, Dutch-Flemish Spinal Cord Society Characteristics, length of stay and functional outcome of patients with spinal cord injury in Dutch and Flemish rehabilitation centres. Spinal Cord 2009; 47: 339-344.

20 Mesard L, Carmody A, Mannarino E, Ruge D. Survival after spinal cord trauma. A life table analysis. Arch Neurol 1978; 35: 78-83.

21 Ronen J, Goldin D, Bluvshtein V, Fishel B, Gelernter I, Catz A. Survival after nontraumatic spinal cord lesions in Israel. Arch Phys Med Rehabil 2004; 85 . 1499-1502.

22 Garshick E, Kelley A, Cohen SA, Garrison A, Gagnon D, Brown R. A prospective assessment of mortality in chronic spinal cord injury. Spinal Cord 2005; 43: 408-416.

23 Van der Woude LH, de Groot S, Postema K, Bussmann JB, Janssen TW ALLRISC et al. Active LifestyLe rehabilitation interventions in aging spinal cord injury (ALLRISC): A multicentre research program. Disabil Rehabil 2013; 35: 1097-1103.

24 DeVivo MJ, Black KJ, Stover SL. Causes of death during the first 12 years after spinal cord injury. Arch Phys Med Rehabil 1993; 74: 248-254.

25 DeVivo MJ, Kartus PL, Stover SL, Rutt RD, Fine PR. Cause of death for patients with spinal cord injuries. Arch Intern Med 1989; 149: 1761-1766. 